\title{
O-C Analysis of Selected 3-Body Systems
}

\section{W. Ogłoza ${ }^{1}$, J. M. Kreiner ${ }^{1}$, G. Stachowski ${ }^{1}$, M. Winiarski ${ }^{1}$,} B. Zakrzewski ${ }^{1}$, S. Doğru ${ }^{2}$ F. Aliçavuş ${ }^{2}$, O. Demircan ${ }^{2}$, and A. Erdem ${ }^{2}$

${ }^{1}$ Mt. Suhora Observatory, Cracow Pedagogical University, 30-084 Cracow, ul Podchorążych 2, Poland email: ogloza@up.krakow.pl

${ }^{2}$ Ulupinar Observatory, Çanakkale Onsekiz Mart University, Turkey

\begin{abstract}
This paper presents the results of the analysis of $(\mathrm{O}-\mathrm{C})$ diagrams of four eclipsing variables. The diagrams are based on times of minima collected in the Cracow database, which contains times of minima found in the literature, from observations at Mt. Suhora and Ulupinar Observatories, or determined using publicly-available photometric surveys (NSVS, ASAS etc).
\end{abstract}

Keywords. stars: binaries: eclipsing, ephemerides

\section{Introduction}

The O-C diagrams were prepared using mainly CCD and photoelectric data. Photographic and visual observations were used only for XZ Per, because of the long timescale of the (O-C) effects. A few scattered minima were rejected because of apparent, unidentified errors. All diagrams clearly show light-time effects due to the influence of a third body.

The commonly-used software written by T. Pribulla was used to determine the orbital parameters of the third body. For each star, (O-C) diagrams were calculated using linear elements, and the data were improved for the average period correction and the residuals of the LTE fit.

\section{Results}

For V1061 Cyg the 3-body effect described by Torres et al. (2006) is confirmed by recent observations.

Table 1. Orbital parameters of 3-body systems

\begin{tabular}{|l|c|c|c|c|}
\hline & BE And & V1061 Cyg & XZ Per & V482 Per \\
\hline P(3) (days) & $3354 \pm 58$ & $5671 \pm 105$ & $24630 \pm 177$ & $6062 \pm 64$ \\
e & $0.62 \pm 0.20$ & $0.43 \pm 0.08$ & $0.61 \pm 0.03$ & $0.84 \pm 0.03$ \\
w(rad) & $5.20 \pm 0.18$ & $4.97 \pm 0.19$ & $3.19 \pm 0.05$ & $3.57 \pm 0.04$ \\
T(periast)(JD) & $2442694 \pm 150$ & $2443058 \pm 178$ & $2400094 \pm 394$ & $2400747 \pm 569$ \\
asin(i) (AU) & $0.82 \pm 0.14$ & $2.46 \pm 0.11$ & $4.23 \pm 0.11$ & $4.83 \pm 0.35$ \\
To(HJD) & 2434962.4035 & 2426355.2335 & 2425150.4482 & 2428327.7615 \\
& \pm 0.0018 & \pm 0.0043 & \pm 0.0009 & \pm 0.0025 \\
P & 0.462902516 & 2.346653857 & 1.15163193 & 2.4467531 \\
(days) & $\pm 4.58 \mathrm{E}-08$ & $\pm 4.03 E-07$ & $\pm 4.18 E-08$ & $\pm 2.49 E-07$ \\
Sum of Sqr & 0.00002 & 0.00091 & 0.0117 & 0.0033 \\
f(m3) & $0.0064 \pm 0.0032$ & $0.0615 \pm 0.0082$ & $0.01665 \pm 0.00137$ & 0.40950 .0893 \\
\hline
\end{tabular}


The period changes of XZ Per were described by Qian (2001) as the result of mass transfer, but recent observations and more complete historic data confirm a 3-body model of the system.

The observed period changes of BD And and V482 Per are currently being analysed.

\section{Database}

The pictures of O-C diagrams and ephemerides based on the last linear trend on the O-C diagrams are presented at: www.as.up.krakow.pl/ephem.

\section{Acknowledgements}

This study was supported by TUBITAK (Scientific and Technological Research Council of Turkey) under Grant No. 108T714. A portion of this study is part of the PhD thesis of Serkan Doğru.

\section{References}

Kreiner, J. M. 2004, AcA, 50, 247

Qian, S. 2001, $A J, 121,1216$

Torres, G., Lacy, C. H., Marschall, L. A., Sheets, H. A., \& Mader, J. A. 2006, ApJ, 640, 1018 